\title{
Updates on Flavor Production from STAR
}

\author{
Sooraj Radhakrishnan ${ }^{1, *}$ \\ ${ }^{1}$ Kent State University, Kent, OH, 44240
}

\begin{abstract}
In these proceedings, some of the latest results from the STAR experiment on heavy, strange and light flavor hadron production and their flow are summarized. Over the years STAR has collected heavy ion collision data over a wide range of collision energies from $\sqrt{s_{\mathrm{NN}}}=200 \mathrm{GeV}$ down to $\sqrt{s_{\mathrm{NN}}}=3 \mathrm{GeV}$. In high energy heavy ion collisions, heavy flavor hadrons are a valuable probe to study properties of the Quark Gluon Plasma (QGP) created in these collisions. Measurements of strange and light flavor hadrons at lower collision energies help understand the properties of the QCD matter at finite baryon densities and the phase structure of the QCD matter. Furthermore, the high statistics data collected at $\sqrt{s_{\mathrm{NN}}}=3 \mathrm{GeV}$ allows measurements of hypernuclei production and flow, which can provide insights into the hyperon - nucleon interactions and hyperon contribution to the nuclear equation of state.
\end{abstract}

\section{Introduction}

Measurements of elliptic flow $\left(v_{2}\right)$ of D mesons and electrons from heavy flavor hadron decays (HFE) in heavy-ion collisions at top RHIC energy and LHC energies show comparable values to those of light flavor hadrons, indicating that charm quarks interact strongly with the Quark Gluon Plasma (QGP) at these energies [1,2]. These measurements have helped constrain the charm quark diffusion coefficient in the QGP [3]. Measurements of HFE $v_{2}$ at lower collision energies can help constrain the temperature dependence of charm quark transport in the QGP. The observed $\mathrm{J} / \psi$ nuclear modification factor $\left(\mathrm{R}_{\mathrm{AA}}\right)$ in heavy-ion collisions at RHIC and LHC has contributions from a regeneration component, in addition to that from in-medium dissociation [4]. Better precision measurements at lower collision energies can also help constrain these different contributions.

The Beam Energy Scan Phase II (BES-II) program at STAR, following the BES-I, aims to conduct high statistics measurements of various observables in the high baryon density region $\left(200<\mu_{B}<720 \mathrm{MeV}\right)$ to understand the phase structure of QCD matter. As part of BES-II, STAR has collected a high statistics dataset of $\mathrm{Au}+\mathrm{Au}$ collisions at $\sqrt{s_{\mathrm{NN}}}=3 \mathrm{GeV}\left(\mu_{B}=720\right.$ $\mathrm{MeV}$ ), using a fixed-target setup. The detailed measurements of the collective flow of various identified light and strange flavor hadrons with this dataset can improve our understanding of the nature and Equation of State (EoS) of the QCD matter in the high $\mu_{B}$ region. In addition, the high statistics $3 \mathrm{GeV}$ dataset allows the measurement of strange and multi-strange hadron production, providing insights into the canonical suppression of strangeness production at low collision energies [6]. The same dataset also enables measurements of the yields, lifetime and flow of hypernuclei, owing to their enhanced cross-sections at these low energies, which can

\footnotetext{
*e-mail: skradhakrishnan@lbl.gov
} 
help understand the hyperon - nucleon interactions and hyperon contribution to the nuclear EoS.

In these proceedings, measurements of HFE $v_{2}$ in $\mathrm{Au}+\mathrm{Au}$ collisions at $\sqrt{s_{\mathrm{NN}}}=54.4$ and $27 \mathrm{GeV}$ and $\mathrm{R}_{\mathrm{AA}}$ of $\mathrm{J} / \psi$ in $\mathrm{Au}+\mathrm{Au}$ collisions at $\sqrt{s_{\mathrm{NN}}}=54.4 \mathrm{GeV}$ are presented. Measurements of $v_{2}$ of identified hadrons, yield ratios of strange and multi-strange hadrons, and yields, lifetime and directed flow $\left(v_{1}\right)$ of hypernuclei ${ }_{3}^{\Lambda} H$ and ${ }_{4}^{\Lambda} H$ in $\mathrm{Au}+\mathrm{Au}$ collisions at $\sqrt{s_{\mathrm{NN}}}=3 \mathrm{GeV}$ are also presented. The results are compared to model calculations and the physics implications are discussed.

\section{Heavy flavor measurements at $\sqrt{s_{\mathrm{NN}}}=54.4$ and $27 \mathrm{GeV}$}

STAR had previously carried out measurements of $v_{2}$ of HFE at $\sqrt{s_{\mathrm{NN}}}=62.4$ and $39 \mathrm{GeV}$ [2]. The measured HFE $v_{2}$ values were found to be smaller, although consistent within the large uncertainties, than the values measured at $200 \mathrm{GeV}$. In 2017 and 2018 STAR collected 10 times more statistics at $\sqrt{s_{\mathrm{NN}}}=54.4 \mathrm{GeV}$ and $27 \mathrm{GeV}$ compared to those used for previous measurements at 62.4 and $39 \mathrm{GeV}$. The left panel of Fig. 1 shows the measured $v_{2}$ of HFE at 54.4 and $27 \mathrm{GeV}$ as a function of transverse momentum $\left(p_{\mathrm{T}}\right)$, compared to the measurements at $200 \mathrm{GeV}$. The measured $v_{2}$ values at $54.4 \mathrm{GeV}$ are non-zero and comparable to those at $200 \mathrm{GeV}$, indicating strong interactions of the charm quarks with the QGP medium. The measurements at $27 \mathrm{GeV}$ show a hint of lower values, albeit with large uncertainties. The right panel of Fig. 1 shows the comparison of the measured HFE $v_{2}$ values at $54.4 \mathrm{GeV}$ to different theoretical model calculations. The TAMU [7] and PHSD [8] model calculations, which incorporate charm quark diffusion in the QGP, describe the data at high $p_{\mathrm{T}}$, but are lower than the measured values at low $p_{\mathrm{T}}$.
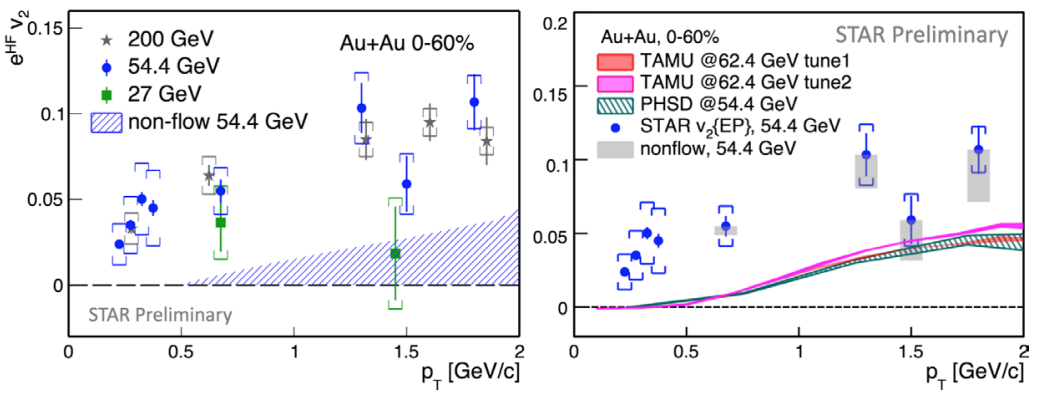

Figure 1. (Left) The $v_{2}$ of HFE as a function of $p_{\mathrm{T}}$ in $0-60 \% \mathrm{Au}+\mathrm{Au}$ collisions compared between $\sqrt{s_{\mathrm{NN}}}$ $=54.4,27$ and $200 \mathrm{GeV}$. The error bars are statistical uncertainties and brackets indicate systematic uncertainties. The blue hatched area indicates the estimated non-flow contribution to the measured $v_{2}$ values. (Right) The $v_{2}$ of HFE as a function of $p_{\mathrm{T}}$ in $0-60 \% \mathrm{Au}+\mathrm{Au}$ collisions at $\sqrt{s_{\mathrm{NN}}}=54.4 \mathrm{GeV}$ compared to different model calculations $[7,8]$.

The in-medium dissociation of $\mathrm{J} / \psi$, due to Debye like color screening in the QGP medium, has been proposed as an evidence of the formation of the QGP and a valuable tool to study its properties, particularly the medium temperature [9]. Suppression of $\mathrm{J} / \psi$ production has been observed at both RHIC and the LHC. However, the observed suppression also gets contribution from regeneration of $\mathrm{J} / \psi$ from deconfined charm quarks in the medium $[4,10]$. Measurements of the $\mathrm{J} / \psi$ suppression at a lower collision energy of $\sqrt{s_{\mathrm{NN}}}=54.4 \mathrm{GeV}$, where the charm quark production cross-section and thus the regeneration contribution are lower, can help better understand the in-medium $\mathrm{J} / \psi$ dissociation. The left panel of Fig. 2 shows the 
nuclear modification factor $\mathrm{R}_{\mathrm{AA}}$, the ratio of yields in $\mathrm{A}+\mathrm{A}$ collisions to that in $\mathrm{p}+\mathrm{p}$ collisions scaled by the number of binary nucleon-nucleon collisions $N_{\text {coll }}$, as a function of number of participating nucleons in the collision $\left(N_{\text {part }}\right)$ for different collision energies. The right panel shows the $\mathrm{R}_{\mathrm{AA}}$ in central collisions as a function of collision energy. The new measurements at $54.4 \mathrm{GeV}$ are shown in red markers, and have significantly improved precision compared to previous low energy STAR measurements at 62.4 and $39 \mathrm{GeV}$ [11]. The measured values show no significant collision energy dependence from 17.2 to $200 \mathrm{GeV}$ within uncertainties. A model calculation [10] that takes into account both in-medium dissociation and regeneration, along with cold nuclear matter effects, can reproduce the measured values.
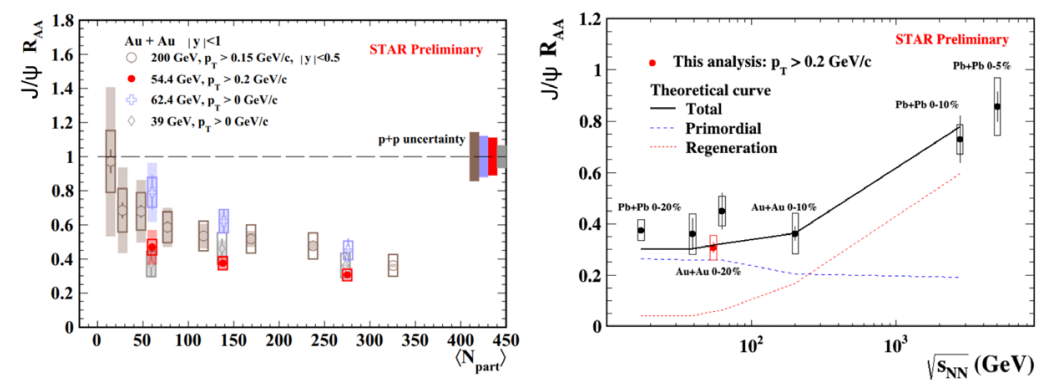

Figure 2. (Left) The $\mathrm{R}_{\mathrm{AA}}$ of $\mathrm{J} / \psi$ as a function of $N_{\text {part }}$ for different collision energies. The error bars, boxes and shaded bands on the data points denote statistical, systematic and $N_{\text {coll }}$ uncertainties respectively. The shaded bands at 1 indicate the uncertainties from the $p+p$ baseline. (Right) The $R_{A A}$ of $J / \psi$ in central collisions as a function of collision energy, including measurements at SPS and LHC [12]. The error bands indicate statistical uncertainties and boxes indicate the combined systematic, $N_{\text {coll }}$ and $\mathrm{p}+\mathrm{p}$ baseline uncertainties.

\section{Strange and light flavor production}

\subsection{Disappearance of partonic collectivity at $\sqrt{s_{\mathrm{NN}}}=3 \mathrm{GeV}$}

The large positive values of $v_{2}$ and its number of constituent quark (NCQ) scaling observed for identified hadrons in high energy heavy-ion collisions have been argued as evidence for the creation of a strongly interacting QGP [13]. At lower collision energies, below $\sqrt{s_{\mathrm{NN}}}=$ $27 \mathrm{GeV}$, where the passage time of the colliding nuclei becomes comparable to or larger than the time scale of parton production, a negative 'squeeze out' contribution from the shadowing by spectator nucleons is expected for the $v_{2}$, in addition to the contribution from partonic collectivity [14]. At even lower collision energies, $\sqrt{s_{\mathrm{NN}}} \sim 5 \mathrm{GeV}$, hadronic transport and baryonic mean field interactions among the colliding nuclei also contribute to the observed flow harmonics [15]. In 2018 STAR collected about $2.6 \times 10^{8}$ minimum bias Au+Au collisions at $\sqrt{s_{\mathrm{NN}}}=3 \mathrm{GeV}$. The high statistics dataset allows to carry out detailed measurements of the $v_{2}$ of various identified hadrons at mid-rapidity in this interesting region of QCD phase space.

Figure 3 shows the NCQ scaled $v_{2}$ of identified hadrons as a function of the scaled transverse kinetic energy in $\mathrm{Au}+\mathrm{Au}$ collisions at $\sqrt{s_{\mathrm{NN}}}=3 \mathrm{GeV}$. The figure also shows measurements at $\sqrt{s_{\mathrm{NN}}}=4.5 \mathrm{GeV}[5]$ and from the new datasets at $\sqrt{s_{\mathrm{NN}}}=54.4$ and $27 \mathrm{GeV}$. The $v_{2}$ values at 54.4 and 27 show NCQ scaling. Positive values and scaling within uncertainties are also observed at $\sqrt{s_{\mathrm{NN}}}=4.5 \mathrm{GeV}$. At $\sqrt{s_{\mathrm{NN}}}=3 \mathrm{GeV}$, the measured $v_{2}$ values turn negative and the NCQ scaling breaks down. Figure 4 shows the centrality dependence of the $v_{2}$ values of different identified hadrons at $\sqrt{s_{\mathrm{NN}}}=3 \mathrm{GeV}$. Calculations from the UrQMD model [16] 
at $\sqrt{s_{\mathrm{NN}}}=3 \mathrm{GeV}$ in the cascade mode, that incorporates hadronic transport and spectator shadowing, and in the meanfield mode, which includes additional baryonic meanfield interactions, are also shown. The UrQMD cascade mode cannot describe the measured $v_{2}$ values and are of opposite sign. The UrQMD meanfield mode calculations, with a value of the incompressibility $\kappa=380 \mathrm{MeV}$, can reproduce the trends in the data, except for $K^{+}$. These measurements and comparisons show that a medium dominated by hadronic and baryonic interactions, rather than partonic interactions, is produced in $\mathrm{Au}+\mathrm{Au}$ collisions at $\sqrt{s_{\mathrm{NN}}}=3$ $\mathrm{GeV}$.

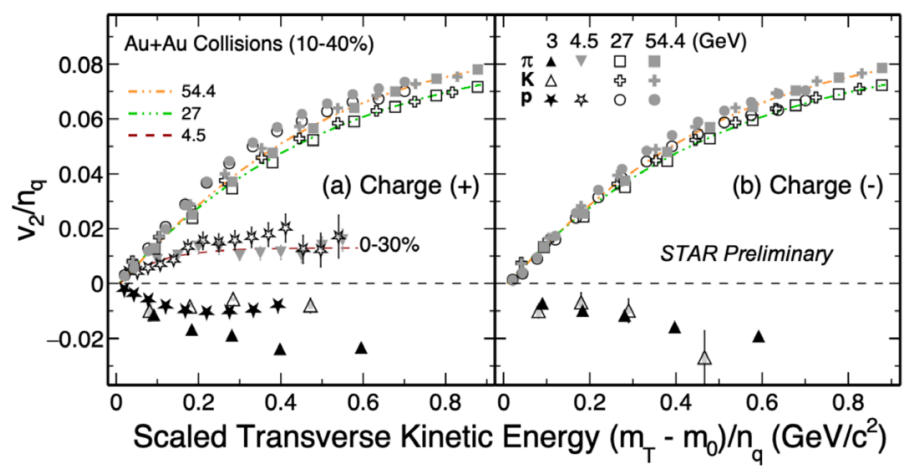

Figure 3. The $v_{2}$ scaled by the number of constituent quarks $n_{q}$ as a function of the scaled transverse kinetic energy at different collision energies for negative (left) and positive particles (right). Statistical and systematic uncertainties are indicated by error bars and shaded bands on data points, respectively. The dashed lines denote the NCQ scaling curves at different energies.

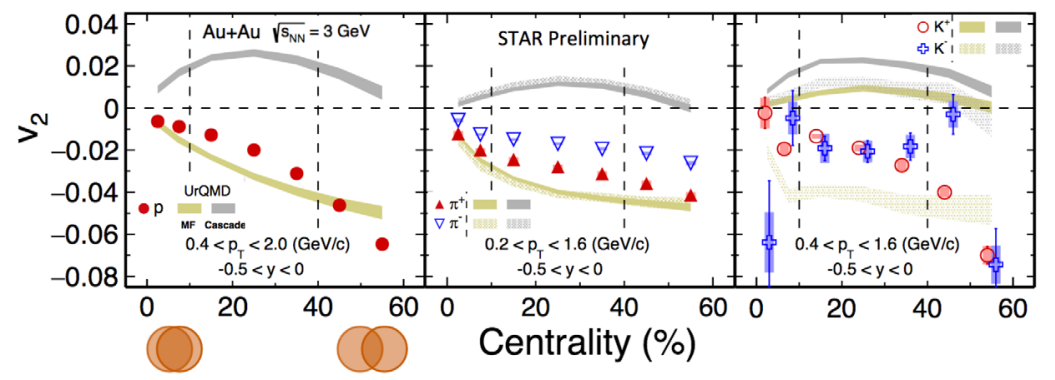

Figure 4. The $v_{2}$ of protons (left), pions (middle) and kaons (right) as a function of centrality for $\mathrm{Au}+\mathrm{Au}$ collisions at $\sqrt{s_{\mathrm{NN}}}=3 \mathrm{GeV}$. Statistical and systematic uncertainties are indicated by error bars and shaded bands on data points, respectively. UrQMD calculations in cascade and meanfiled modes are shown as gray and golden bands respectively.

\subsection{Canonical suppression of strangeness production}

Statistical thermal models have been successful in describing the hadron yields in heavy-ion collisions [17]. At low collision energies and in small collision systems, as the strangeness production is rare, it has been argued that the strangeness number needs to conserved locally and on an event by event basis. Therefore a Canonical Ensemble (CE) is more suitable to describe the statistical production of strange hadrons as opposed to a Grand Canonical Ensemble (GCE). This would cause progressive suppression of yields of strange hadrons with non-zero strangeness numbers $[6,18]$. Figure 5 shows the yield ratios of $\phi$ mesons to that of $K^{-}$on the 
left and yield ratios of $\phi$ relative to $\Xi^{-}$on the right as a function of collision energy. The new measurements at $3 \mathrm{GeV}$ are shown as colored markers. The GCE calculations are strongly disfavored, being lower than the measured ratios at $5 \sigma$ and $4 \sigma$ significances respectively, in the $0-10 \%$ most central collisions. The CE calculation with a strangeness correlation length $\left(r_{c}\right) \sim 3.2 \mathrm{fm}$ can describe the $\phi / K^{-}$yield ratio. There is a hint of a centrality dependence of the ratios at $\sqrt{s_{\mathrm{NN}}}=3 \mathrm{GeV}$. Future higher precision measurements can provide better insights into the centrality dependence. Transport model calculations from a modified UrQMD [19] and SMASH [20] that take into account high mass resonances that decay into $\phi$ and $\Xi^{-}$can also describe the ratios at $3 \mathrm{GeV}$.
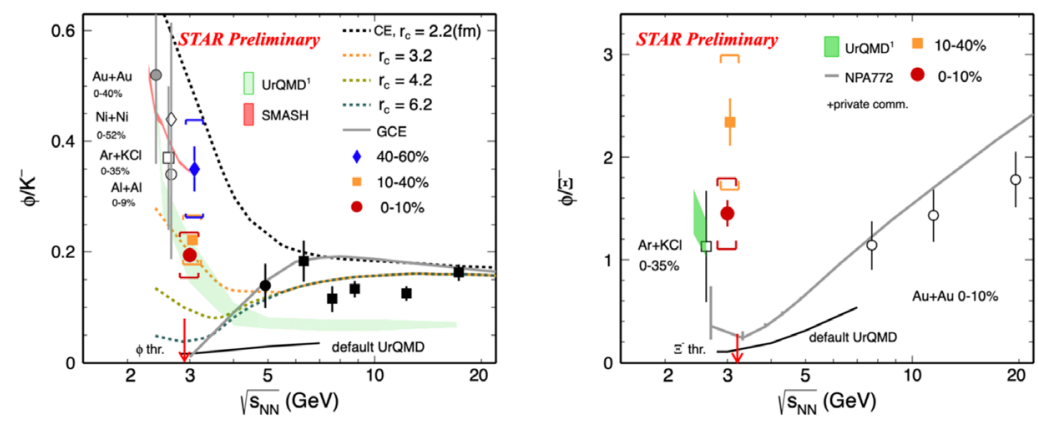

Figure 5. The yield ratios of $\phi$ to that of $K^{-}$(left) and of $\phi$ to that of $\Xi^{-}$(right) as a function of collision energy. The new measurements at $3 \mathrm{GeV}$ are shown in colored markers and at other energies [21] in solid black and open gray markers. The error bars and brackets on the $3 \mathrm{GeV}$ data points denote the statistical and systematic uncertainties respectively. The gray solid line indicates the GCE calculation while the dashed lines indicate $\mathrm{CE}$ calculations with different $r_{c}$ values [6, 18, 22]. The default UrQMD [16] calculations are shown in solid black line, while the modified UrQMD [19] and SMASH [20] calculations are shown in the green and red bands respectively.

\section{Hypernuclei production at $\sqrt{s_{\mathrm{NN}}}=3 \mathrm{GeV}$}

Hypernuclei production cross-sections in heavy-ion collisions are large at colliding energies $\sim 3 \mathrm{GeV}$ [23]. This provides an opportunity to measure the lifetime, yield and collective flow of hypernuclei with the $\sqrt{s_{\mathrm{NN}}}=3 \mathrm{GeV} \mathrm{Au}+$ Au collision dataset. The lifetimes of ${ }_{3}^{\Lambda} H$ and ${ }_{4}^{\Lambda} H$ measured using the $3 \mathrm{GeV}$ dataset are $232 \pm 29$ (stat) \pm 37 (sys) and $218 \pm 8$ (stat) \pm 12 (sys), respectively. The ${ }_{4}^{\Lambda} H$ lifetime measurement obtained is the most precise to date. The two left panels of Fig. 6 show the yields within rapidity $|y|<0.5$ of the hypernuclei ${ }_{3}^{\Lambda} H$ and ${ }_{4}^{\Lambda} H$, along with model calculations, as a function of collision energy. Thermal model calculations [23] can describe the measured ${ }_{3}^{\Lambda} H$ yield at $3 \mathrm{GeV}$ and also at the LHC energy [24]. The thermal model calculations are done with a $\mathrm{CE}$ for strangeness production. The model however underpredicts the yield of ${ }_{4}^{\Lambda} H$ at $3 \mathrm{GeV}$. Coalescence model calculations [25] overestimate the ${ }_{3}^{\Lambda} H$ yield while under-predict the ${ }_{4}^{\Lambda} H$ yield at $3 \mathrm{GeV}$. A hybrid UrQMD calculation over-predicts yields of both ${ }_{3}^{\Lambda} H$ and ${ }_{4}^{\Lambda} H$. The right panel of Fig. 6 shows the slope of directed flow at midrapidty, $d v_{1} /\left.d y\right|_{y=0}$, of various light nuclei and hypernuclei as a function of their mass for $\mathrm{Au}+\mathrm{Au}$ collisions at $\sqrt{s_{\mathrm{NN}}}=3 \mathrm{GeV}$. A mass scaling of directed flow slope has been observed for light nuclei which is attributed to the coalescence production of light nuclei [26]. The hypernuclei also show a mass scaling of $v_{1}$ slope, albeit with large uncertainties, suggesting coalescence production also for hypernuclei. These measurements will help better constrain the hypernucleus production models and also the hyperon contribution to nuclear EoS. 

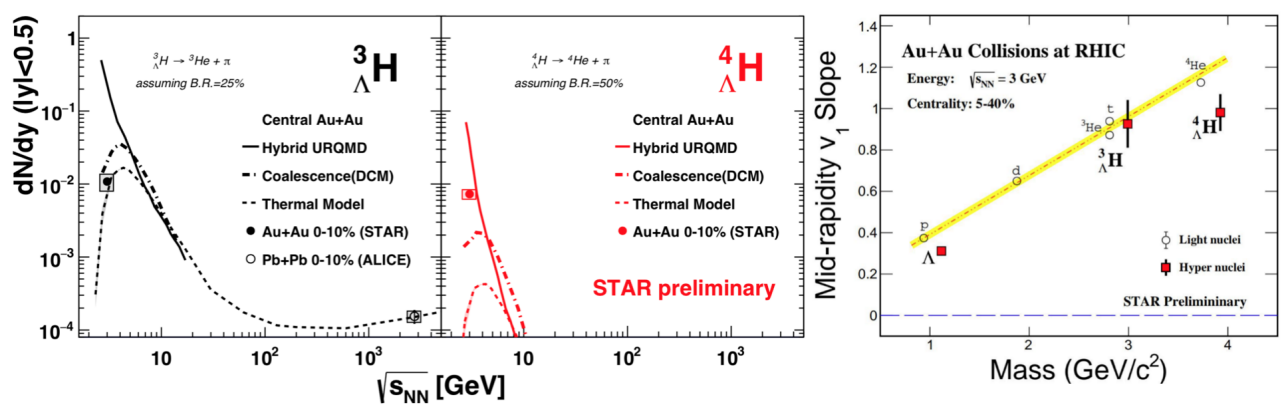

Figure 6. The yield in $|y|<0.5$ of ${ }_{3}^{\Lambda} H$ (left) and ${ }_{4}^{\Lambda} H$ (middle), along with model comparisons, as a function of collision energy. The boxes indicate the total uncertainties. (Right) The measured slope, $d v_{1} /\left.d y\right|_{y=0}$, in $\mathrm{Au}+\mathrm{Au}$ collisions at $\sqrt{s_{\mathrm{NN}}}=3$ of different light nuclei and hypernuclei as a function of mass. Error bars indicate total uncertainties. The yellow line shows a linear fit to the light nuclei measurements. Combined results from the 2-body and 3-body decay channels are used for the ${ }_{3}^{\Lambda} H$ $v_{1}$ measurements, while for ${ }_{4}^{\Lambda} H v_{1}$ measurements and for yield measurements of both the hypernuclei results from only 2-body decay channel are used.

\section{Summary}

We have presented some of the newest measurements from STAR on heavy, strange and light flavor hadron production and collective flow. Measurement of HFE $v_{2}$ with a much improved precision in $\mathrm{Au}+\mathrm{Au}$ collisions at $\sqrt{s_{\mathrm{NN}}}=54.4 \mathrm{GeV}$, compared to previous measurements at 62.4 and $39 \mathrm{GeV}$, shows non-zero values comparable to the $v_{2}$ of HFE measured at $200 \mathrm{GeV}$. This indicates that charm quarks interact strongly with the QGP medium produced in 54.4 $\mathrm{GeV}$ collisions. The $\mathrm{R}_{\mathrm{AA}}$ of $\mathrm{J} / \psi$ measured in $\mathrm{Au}+\mathrm{Au}$ collisions at $\sqrt{s_{\mathrm{NN}}}=54.4 \mathrm{GeV}$ has improved precision compared to previous measurements at 62.4 and $39 \mathrm{GeV}$. The measurements show no significant collision energy dependence of $\mathrm{R}_{\mathrm{AA}}$ within uncertainties compared to that at $200 \mathrm{GeV}$ and other lower energy measurements. Elliptic flows of identified hadrons in $\mathrm{Au}+\mathrm{Au}$ collisions at $\sqrt{s_{\mathrm{NN}}}=3 \mathrm{GeV}$ show negative values and a breakdown of the NCQ scaling. Hadronic transport model calculations from UrQMD with baryonic meanfield interactions can describe the trends seen in data. These observations indicate the formation of a medium dominated by hadronic interactions and a nuclear EoS in $\mathrm{Au}+\mathrm{Au}$ collisions at $3 \mathrm{GeV}$. Strange and multi-strange hadron yield measurements in $\mathrm{Au}+\mathrm{Au}$ collisions at $\sqrt{s_{\mathrm{NN}}}$ $=3 \mathrm{GeV}$ strongly disfavor GCE calculations and show evidence of canonical suppression in strangeness production. Thermal model calculations are consistent with the measured ${ }_{3}^{\Lambda} \mathrm{H}$ yield, while under-predct the ${ }_{4}^{\Lambda} H$ yield. Coalescence model calculations fail to describe yields of both the hypernuclei. Measurements of directed flow slope, $d v_{1} /\left.d y\right|_{y=0}$, of light nuclei and hypernuclei show mass scaling within uncertainties. The mass scaling is consistent with a picture of coalescence production for the light nuclei and hypernuclei.

\section{References}

[1] L. Adamczyk et al. (STAR), Phys. Rev. Lett. 118, 212301 (2017); S. Acharya et al. (ALICE), Phys. Rev. Lett. 120, 102301 (2018)

[2] L. Adamczyk et al. (STAR), Phys. Rev. C 95, 034907 (2017)

[3] X. Dong, Y.-J. Lee, R. Rapp, Ann. Rev. Nucl. Part. Sci. 69 417-445 (2019) 
[4] B. Abelev et. al (ALICE), Phys. Lett. B 734, 314-327 (2014); J. Adam et al. (STAR), Phys. Lett. B 797, 134917 (2019)

[5] J. Adam et al. (STAR), Phys. Rev. C 10334908 (2021)

[6] K. Redlich and A. Tounsi, Eur. Phys. J. C 24, 589 (2002)

[7] M. He et al., Phys. Rev. C 91, 024904 (2015)

[8] T. Song et al., Phys. Rev. C 92, 014910 (2015); T. Song et al., Phys. Rev. C. 96, 014905 (2017)

[9] T. Matsui, H. Satz, Phys. Lett. B 178 416-422 (1986).

[10] X. Zhao, R. Rapp, Phys. Rev. C 82, 064905 (2010)

[11] L. Adamczyk et al. (STAR), Phys. Lett. B 771, 13 (2017)

[12] L. Kluberg, Eur. Phys. J. C 43, 145 (2005); B. Abelev et. al (ALICE), Phys. Lett. B 734, 314 (2014); X. Bai (for ALICE Collaboration), Nucl. Phys. A 1005, 121769 (2021)

[13] U. Heinz and R. Snellings, Annu. Rev. Nucl. Part. Sci. 63123 (2013); B. I. Abelev et al. (STAR), Phys. Rev. Lett. 99, 112301 (2007)

[14] C. Pinkenburget et al.(E895), Phys. Rev. Lett. 83, 1295 (1999)

[15] P. Danielewicz et al, Science 298, 1592-1596, (2002)

[16] M. Bleicheret et al., J. Phys. G 25, 1859 (1999)

[17] A Andronic et al. Nature 561, 321 (2018)

[18] A. Andronic et al. Nucl. Phys. A 772 167-199 (2006)

[19] J. Steinheimer and M. Bleicher, J. Phys. G: Nucl. Part. Phys. 43, 015104 (2015).

[20] V. Steinberg et al., Phys. Rev. C 99, 064908 (2019)

[21] M. S. Abdallah et al. (STAR), arXiv:2108.00924 (2021)

[22] S. Wheaton et al., Comput. Phys. Commun. 180, 84 (2009)

[23] A. Andronic et al., Phys. Lett. B 697, 203 (2011)

[24] J. Adam et al. (ALICE), Phys. Lett. B 754, 360 (2016)

[25] J. Steinheimer et al. Phys. Lett. B 714, 47985 (2012)

[26] L. Adamczyk et al. (STAR), Phys. Rev. C 94, 034908 (2016) 